\title{
Acoustic transmission line metamaterial with negative/zero/positive refractive index
}

\author{
Frédéric Bongard, ${ }^{*}$ Hervé Lissek, ${ }^{\dagger}$ and Juan R. Mosig \\ Laboratory of Electromagnetics and Acoustics (LEMA), Ecole Polytechnique Fédérale de Lausanne (EPFL),
} CH-1015 Lausanne, Switzerland

(Received 30 November 2009; revised manuscript received 20 August 2010; published 16 September 2010)

\begin{abstract}
A one-dimensional acoustic negative refractive index metamaterial based on the transmission line approach is presented. This structure implements the dual transmission line concept extensively investigated in microwave engineering. It consists of an acoustic waveguide periodically loaded with membranes realizing the function of series "capacitances" and transversally connected open channels realizing shunt "inductances." Transmission line based metamaterials can exhibit a negative refractive index without relying on resonance phenomena, which results in a bandwidth of operation much broader than that observed in resonant devices. In the present case, the negative refractive index band extends over almost one octave, from 0.6 to $1 \mathrm{kHz}$. The developed structure also exhibits a seamless transition between the negative and positive refractive index bands with a zero index at the transition frequency of $1 \mathrm{kHz}$. At this frequency, the unit cell is only one tenth of the wavelength. Simple acoustic circuit models are introduced, which allow efficient designs both in terms of dispersion and impedance, while accurately describing all the physical phenomena. Using this approach, a good matching at the structure terminations is achieved. Full-wave simulations, made for a 10-cell-long structure, confirm the good performances in terms of dispersion diagram, Bloch impedance, and reflection and transmission coefficients.
\end{abstract}

DOI: 10.1103/PhysRevB.82.094306

PACS number(s): 43.20.+g, 43.35.+d

\section{INTRODUCTION}

Metamaterials are broadly defined as artificial composite materials specifically engineered to produce desired unusual properties not readily available in nature. This research area has been first extensively developed for electromagnetic waves, resulting, for instance, in structures with simultaneous negative permittivity and permeability, thereby enabling new intriguing phenomena such as negative refraction. ${ }^{1,2}$ Recently, the analogs of such structures for acoustic and elastic waves have received considerable interest. The resulting acoustic (respectively, elastic) metamaterials are composite structures obtained by loading a host fluid medium (respectively, elastic solid) with subwavelength elastic and/or fluid inclusions, for which effective parameters such as the mass density and the bulk modulus can be defined and possibly take negative values. When these two parameters are simultaneously negative, negative acoustic refraction and subwavelength focusing can be achieved, ${ }^{3}$ as reported in electromagnetics for the perfect lens proposed by Pendry. 4

It is worth noticing that negative acoustic refraction can also be achieved with phononic crystals from band-folding effects due to Bragg scattering ${ }^{5-9}$ but some distinctions are usually drawn. On one side, phononic crystals are operated in a frequency range where the lattice constant is on the order of half a wavelength or more, which prevents an effective-medium description. On the other hand, metamaterials comprise subwavelength unit cells and can be thus ascribed effective-medium parameters. . $^{3,10,11}$

Most of the acoustic metamaterials with negative parameters reported to date are based on localized resonances in the unit cell, hence the name "locally resonant sonic material" (or "locally resonant phononic crystal"). ${ }^{10}$ From a general perspective, the particles forming the composite material should exhibit monopolar and/or dipolar resonances in order to give rise to a negative bulk modulus and/or a negative mass density, respectively, in a small band above the resonance frequency. ${ }^{12,13}$

Various structures have been proposed to achieve negative parameters for acoustic and elastic metamaterials. For instance, an elastic metamaterial with negative effective-mass density was achieved with an array of hard spheres coated with a soft cladding embedded in a stiff host medium. ${ }^{13-15}$ Similar effects have been observed for coated cylinders in a fluid medium (acoustic metamaterial). ${ }^{16}$ A negative bulk modulus was achieved for an elastic metamaterial with an array of bubble-contained-water spheres, ${ }^{13}$ and for acoustic metamaterials with an array of subwavelength Helmholtz resonators, ${ }^{17-19}$ as well as with Helmholtz-type resonators with slits ${ }^{20}$ or side holes in a host waveguide. ${ }^{21}$ Similar structures had also been previously investigated in a different context, such as in Ref. 22 where the purpose was to achieve spectral gaps.

However, only a few double negative acoustic and elastic media have been reported to date. A crystal consisting of soft rubber spheres in water was reported as a double negative acoustic medium, ${ }^{12}$ for which both the monopolar and dipolar resonances required for negative parameters were obtained from the same particle. This approach has been criticized in Ref. 13, where the use of two distinct resonance mechanisms (bubble-contained-water spheres and rubbercoated-gold spheres) has rather been proposed to achieve double negativity, in this specific case for an elastic metamaterial.

In the realm of electromagnetics, there is a common distinction between two types of metamaterials: arrays of resonant inclusions, such as the split-ring resonator and wire medium, ${ }^{23}$ and transmission line (TL) based metamaterials. ${ }^{2,24-26}$ While the materials of the first kind are inherently narrow band and lossy due to their resonant na- 
ture, the latter can exhibit the desired metaproperties, such as negative refraction, over a much larger bandwidth and with lower losses since they do not explicitly rely on resonance phenomena. It appears that most of the acoustic metamaterials reported to date belong to the category of resonant inclusions, whereas very few works on the acoustic counterparts of TL-based metamaterials have been reported. In this work, we fill in this gap by proposing a negative refractive index acoustic metamaterial based on the dual TL concept. This requires the realization of acoustic or mechanical elements which implement shunt "inductances" (i.e., acoustic masses) and series "capacitances" (i.e., acoustic compliances). Preliminary works have shown how to achieve shunt acoustic masses with short tubes connected to a host waveguide, resulting in a metamaterial with negative bulk modulus, ${ }^{21}$ but clear guidelines on how to realize a series compliance in such a system are still missing.

This paper presents a possible realization of acoustic dual TL in which the series compliances are realized with membranes and the shunt masses with short open channels. To introduce and develop these concepts we make an extensive use of circuit modeling, which is shown to be a powerful tool for both understanding and design purposes.

Recent publications, found during the review process, report the use of thin membranes to achieve a medium with negative effective density, ${ }^{27}$ as well as the combination of membranes and side holes to achieve a negative index medium. ${ }^{28}$ The experimental demonstrations reported in these references support the theoretical model and the numerical results presented in this paper. Thanks to our comprehensive approach, we are able to propose an improved design which exhibits not only a negative index band, but also a zero index frequency with nonzero group velocity and a good impedance matching. A time dependence in $\exp ($ $+j \omega t)$ is assumed in all the paper.

\section{TL MODELING OF CONVENTIONAL AND METAMATERIALS}

TLs are circuit-based concepts that can be used to describe the propagation of waves in different systems. This section describes the adopted conventions associated with this formalism when used for acoustic waves in a fluid and introduces the concept of TL-based metamaterial. In the acoustic circuit modeling used in this work, the voltage corresponds to the acoustic pressure $p$ and the current to the volume velocity $q$ flowing through a surface $S$. This representation is often used for waveguide related problems, where $S$ is naturally the waveguide cross-sectional area, ${ }^{29,30}$ but it can also be applied to plane waves in an unbounded medium, in which case $S$ can be chosen arbitrarily.

\section{A. Conventional materials}

With the adopted convention, an incremental section $d z$ of a conventional fluid can be described by the model of Fig. 1(a), where $m_{\mathrm{a}}=(\rho / S) d z$ is an acoustic mass, or inertance $\left[\right.$ in $\left.\left(\mathrm{kg} / \mathrm{m}^{4}\right)\right]$, and $C_{\mathrm{a}}=(S / K) d z$ is an acoustic compliance $\left[\right.$ in $\left.\left(\mathrm{m}^{3} / \mathrm{Pa}\right)\right]$, and $\rho$ and $K$ are the density and bulk

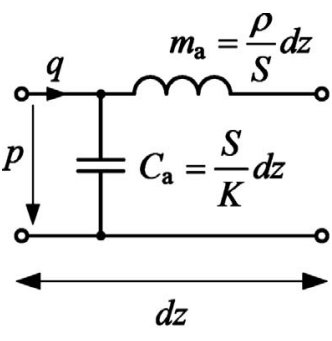

(a)

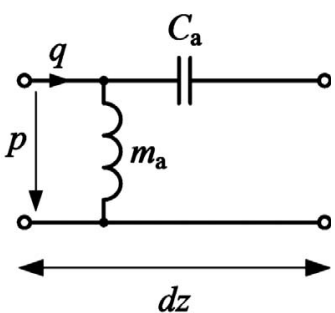

(b)
FIG. 1. Incremental circuit for (a) a conventional medium and (b) a dual medium exhibiting a negative refractive index.

modulus of the medium, respectively. The physical equations corresponding to the adopted TL representation are

$$
\frac{d p}{d z}=-j \omega \frac{\rho}{S} q \quad \text { and } \quad \frac{d q}{d z}=-j \omega \frac{S}{K} p .
$$

The characteristic impedance of the TL whose incremental section is the one of Fig. 1(a) is given by $Z_{\mathrm{ac}}=Z_{\mathrm{c}} / S$ [in $\left.\left(\Omega_{\mathrm{a}} \equiv \mathrm{Pa} \mathrm{s} / \mathrm{m}^{3}\right)\right]$, where $Z_{\mathrm{c}}=\sqrt{\rho K}$ is the characteristic acoustic impedance of the considered medium. The corresponding wave velocity is $c=\sqrt{K / \rho}$ and the wave vector is defined as $k=\omega / c$. In this work, the considered medium is air with the parameters $\rho=1.188 \mathrm{~kg} / \mathrm{m}^{3}, K=137.4 \mathrm{kPa}$, $Z_{\mathrm{c}}=404 \mathrm{~Pa} \mathrm{~s} / \mathrm{m}$, and $c=340 \mathrm{~m} / \mathrm{s}^{31}$

\section{B. Dual topology}

Figure 1(b) shows the dual topology of the conventional TL, which is often referred to as the dual TL. Such a structure is known to exhibit a negative refractive index over an infinite bandwidth. ${ }^{2,25}$ However, the dual TL cannot be implemented in practice in a fully distributed manner but must be realized by periodically loading a host medium with discrete shunt acoustic masses and series acoustic compliances. Considering the natural contribution of the nonvanishing connections between these elements, the resulting periodic structure unit cell is the one shown in Fig. 2. At low frequency, the response is dominated by $m_{\mathrm{ap}}$ and $C_{\mathrm{as}}$, resulting in a left-handed (LH) behavior ( $\equiv$ negative refractive index), whereas $m_{\text {as }}$ and $C_{\text {ap }}$ are predominant at higher frequency, which then results in a right-handed $(\mathrm{RH})$ behavior (三positive refractive index). In microwave engineering, interesting applications exist where both of these bands are used, which is why this structure has been named the composite right/left-handed transmission line (CRLH TL). ${ }^{24,25}$

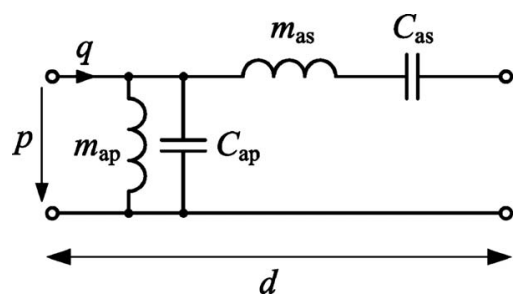

FIG. 2. Unit cell of the CRLH TL, a particular type of TL-based metamaterial. The lattice constant is referred to as $d$. 


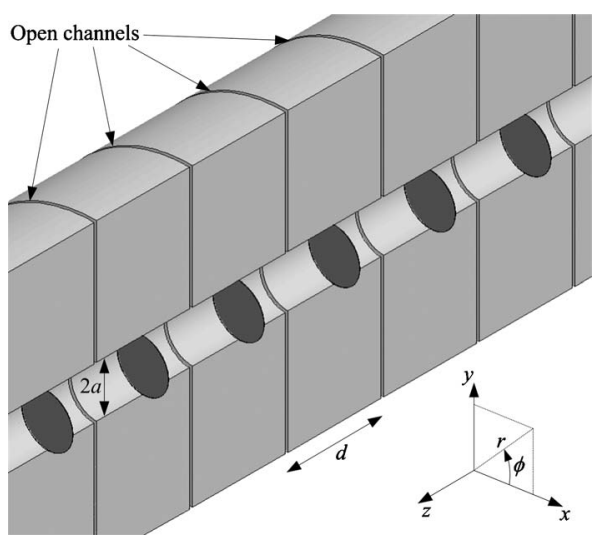

FIG. 3. The proposed CRLH TL combining membranes (in dark) and radial open channels. The main pipe has been cut in the $y z$ plane for visibility. Due to the perfect symmetry of revolution, all the relevant field quantities only depend on the longitudinal and transverse coordinates $z$ and $r$, respectively.

An interesting particularity of this structure is that a seamless transition (i.e., without band gap) between the $\mathrm{LH}$ and $\mathrm{RH}$ bands can be achieved under the so-called balanced condition. ${ }^{25}$ The goal of this work is precisely to synthesize a structure that implements the circuit of Fig. 2 for acoustic waves.

It should be stressed here that the other types of acoustic metamaterials reported in Sec. I can also be described by circuit and TL concepts. However, the main characteristic of TL-based metamaterials such as those based on the dual TL concept (or more generally the CRLH TL) is that they exhibit the desired metaproperties without explicitly relying on resonance phenomena.

Finally, it must be pointed out that the circuits in Figs. 1 and 2 are lossless and hence correspond to ideal structures made up with lossless materials. In practice, losses could be represented by small equivalent resistances in the equivalent circuit. However, since as already mentioned, losses can be very small in TL-based metamaterials, their equivalent circuits can be assumed lossless in a first analysis.

\section{DESCRIPTION OF THE PROPOSED STRUCTURE}

The CRLH metamaterial implementation proposed in this work is shown in Fig. 3. The host "medium" is an acoustic waveguide with circular cross section and perfectly rigid walls operated in its dominant mode region (plane waves). The realization of series compliances using only acoustic elements is not obvious. Here, we propose to use mechanical elements consisting of membranes, for which the restoring force provides the required series compliance. It can be mentioned that membranes have already been proposed in a metamaterial context as a mean to achieve negative dynamic mass by operating them between two eigenmodes. ${ }^{32}$ Here, the membranes are rather operated below and around their first resonance.

Shunt acoustic masses can be simply achieved with transversally connected open channels. ${ }^{30}$ Based on this concept, the solution adopted here consists of radial channels operated

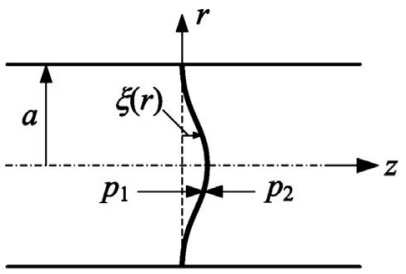

(a)

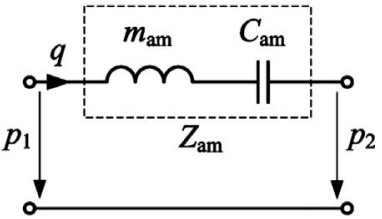

(b)
FIG. 4. Elastic circular membrane clamped to an acoustic waveguide. (a) Cut view. (b) Equivalent acoustic circuit.

in the main waveguide structure, as shown in Fig. 3. A detailed analysis of the two elements introduced above is provided in Secs. III A and III B, respectively.

\section{A. Realization of series compliances with membranes}

\section{Description}

The considered element is a circular membrane clamped at its perimeter to a host waveguide, as illustrated in Fig. 4(a). It is characterized by its Young's modulus E, Poisson's ratio $\nu$, mass density $\rho_{\mathrm{m}}$, thickness $h$, and radius $a$, which is also the radius of the waveguide. The surface of the membrane is $S=\pi a^{2}$. No tension is applied to the membrane.

\section{Theoretical modeling}

The membrane is modeled as a thin plate described by the transverse displacement $\xi(r)$, which satisfies the flexural waves equation $29,33,34$

$$
\nabla^{4} \xi-k_{\mathrm{m}}^{4} \xi=\frac{\Delta p}{D} \quad \text { with } k_{\mathrm{m}}^{2}=\omega \sqrt{\frac{\rho_{\mathrm{m}}^{\prime \prime}}{D}}
$$

and where $\rho_{\mathrm{m}}^{\prime \prime}=\rho_{\mathrm{m}} h$ is the surface mass density of the plate and $D$ designates its flexural rigidity, which is given by ${ }^{34}$

$$
D=\frac{E h^{3}}{12\left(1-\nu^{2}\right)} .
$$

In Eq. (2), $\Delta p$ represents the source term, which corresponds to the net pressure on the plate. It can be written as $\Delta p=p_{1}-p_{2}$, where $p_{1}$ and $p_{2}$ are the acoustic pressure on the two faces, as suggested in Fig. 4(a). The general solution of Eq. (2) in polar coordinates is derived in Ref. 34; only the result is recalled here. Assuming a uniform pressure distribution over the plate, which is verified in case of plane-wave incidence and small displacements, and considering only axially symmetrical modes, the following general solution for $\xi(r)$ is obtained,

$$
\xi(r)=-\frac{\Delta p}{k_{\mathrm{m}}^{4} D}+A J_{0}\left(k_{\mathrm{m}} r\right)+B I_{0}\left(k_{\mathrm{m}} r\right),
$$

where $J_{n}$ and $I_{n}$ are the regular and modified Bessel's functions of the first kind of order $n$, respectively. The application of the boundary conditions corresponding to a clamped membrane $\left[\xi(r=a)=0,\left.\frac{d \xi}{d r}\right|_{r=a}=0\right]$ to Eq. (4) yields the two constants, 


$$
\begin{aligned}
& A=\frac{\Delta p}{k_{\mathrm{m}}^{4} D} \cdot \frac{I_{1}\left(k_{\mathrm{m}} a\right)}{J_{0}\left(k_{\mathrm{m}} a\right) I_{1}\left(k_{\mathrm{m}} a\right)+J_{1}\left(k_{\mathrm{m}} a\right) I_{0}\left(k_{\mathrm{m}} a\right)}, \\
& B=\frac{\Delta p}{k_{\mathrm{m}}^{4} D} \cdot \frac{J_{1}\left(k_{\mathrm{m}} a\right)}{J_{0}\left(k_{\mathrm{m}} a\right) I_{1}\left(k_{\mathrm{m}} a\right)+J_{1}\left(k_{\mathrm{m}} a\right) I_{0}\left(k_{\mathrm{m}} a\right)} .
\end{aligned}
$$

The dynamic response of the plate to the resulting force can be represented by the mechanical impedance $Z_{\mathrm{m}}$, defined as a ratio force over velocity as follows: ${ }^{34}$

$$
Z_{\mathrm{m}}=\frac{\iint_{S} \Delta p(r) d S}{j \omega \bar{\xi}},
$$

where $\bar{\xi}=(1 / S) \iint_{S} \xi(r) d S$ is the mean transverse displacement over the plate surface. Assuming a uniform pressure distribution over the plate and using Eqs. (4) and (5), the mechanical impedance $Z_{\mathrm{m}}$ becomes ${ }^{34}$

$$
Z_{\mathrm{m}}=-j \omega m \cdot \frac{I_{1}\left(k_{\mathrm{m}} a\right) J_{0}\left(k_{\mathrm{m}} a\right)+J_{1}\left(k_{\mathrm{m}} a\right) I_{0}\left(k_{\mathrm{m}} a\right)}{I_{1}\left(k_{\mathrm{m}} a\right) J_{2}\left(k_{\mathrm{m}} a\right)-J_{1}\left(k_{\mathrm{m}} a\right) I_{2}\left(k_{\mathrm{m}} a\right)},
$$

where $m=\rho_{\mathrm{m}}^{\prime \prime} S=\rho_{\mathrm{m}} S h$ is the mass of the plate.

Inside the acoustic waveguide, the elastic membrane can be actuated by an incident acoustic field. The fluid pressure represents a load on the membrane and the membrane in turn provides an acceleration to the surrounding fluid. In such acoustic-structure interactions, at any point on the membrane the normal component of the acoustic velocity at both sides is continuous across the membrane and is equal to the transverse velocity of the membrane $v_{\xi}=j \omega \xi$. The volume velocity $q$ generated by the pressure difference between the two sides of the membrane is given by

$$
q=\iint_{S} v_{\xi}(r) d S=\bar{v}_{\xi} S=j \omega \bar{\xi} S .
$$

Assuming a uniform pressure field on the membrane, the effect of the latter on the acoustic field can be represented by the acoustic impedance $Z_{\mathrm{am}}$ defined as ${ }^{29,34}$

$$
Z_{\mathrm{am}}=\frac{\Delta p}{q}=\frac{Z_{\mathrm{m}}}{S^{2}},
$$

with $Z_{\mathrm{m}}$ defined in Eq. (6) and expressed in Eq. (7). As a result, the equivalent acoustic circuit of a membrane in an acoustic waveguide is a series impedance $Z_{\mathrm{am}}$ loading the TL representing the waveguide, as shown in Fig. 4(b). Indeed, the membrane introduces a discontinuity in the acoustic pressure field, but the normal component of the acoustic velocity, and thus the volume velocity $q$, is continuous across the membrane.

At low frequency up to the first resonance of this dynamic system, which is given by ${ }^{33}$

$$
f_{\mathrm{r}}=1.6259 \frac{1}{a^{2}} \sqrt{\frac{D}{\rho_{\mathrm{m}}^{\prime \prime}}}=0.4694 \frac{h}{a^{2}} \sqrt{\frac{E}{\rho_{\mathrm{m}}\left(1-\nu^{2}\right)}},
$$

the impedance given by Eqs. (7) and (9) can be approximated with very good accuracy by a series resonant circuit

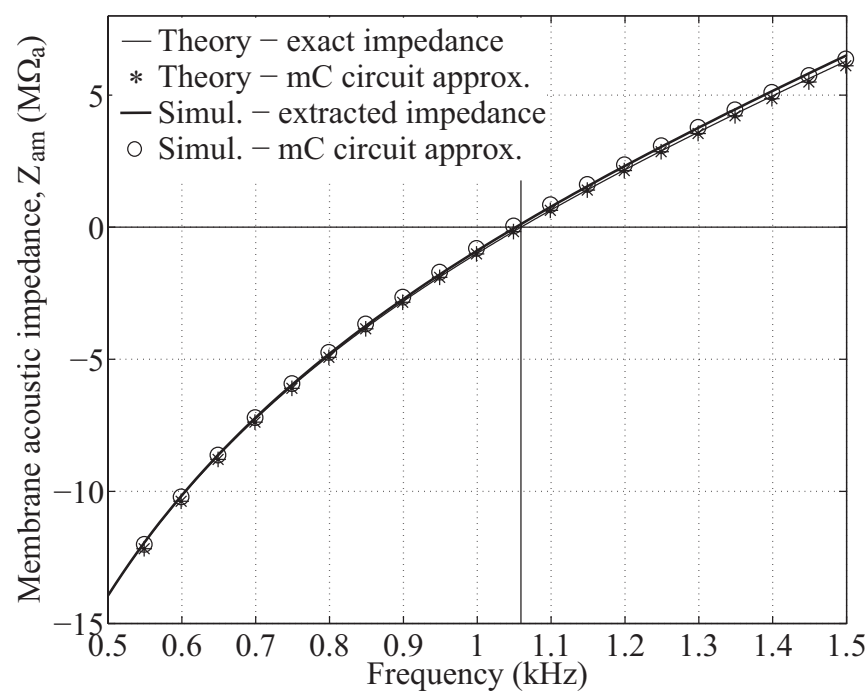

FIG. 5. Acoustic impedance (imaginary part) of the considered membrane. The "Theory-exact impedance" curve corresponds to Eq. (9) [using Eq. (7)], the "Simul.—extracted impedance" curve is the impedance directly extracted from simulation results, and the two "mC circuit approx." curves correspond to the circuit of Fig. 4(b) with the parameters given in Table I.

comprising an acoustic mass $m_{\mathrm{am}}$ and a compliance $C_{\mathrm{am}}$, as shown in Fig. 4(b). The values of these two elements are calculated such that the resulting impedance fits the original impedance and its frequency derivative at the first resonance $f_{\mathrm{r}}$, leading to the following expressions:

$$
m_{\mathrm{am}}=1.8830 \frac{\rho_{\mathrm{m}} h}{\pi a^{2}} \text { and } C_{\mathrm{am}}=\frac{\pi a^{6}}{196.51 D} .
$$

\section{Validation}

In order to validate the presented theoretical modeling, we consider a membrane made of DuPont ${ }^{\mathrm{TM}}$ Kapton ${ }^{\circledR}$ FPC $\left(E=2.758 \mathrm{GPa}, \nu=0.34\right.$, and $\left.\rho_{\mathrm{m}}=1420 \mathrm{~kg} / \mathrm{m}^{3}\right)$ of thickness $h=125 \mu \mathrm{m}$ and radius $a=9.06 \mathrm{~mm})$. This membrane-loaded waveguide has been simulated with COMSOL MULTIPHYSICS and characterized in terms of reflection and transmission coefficients under plane-wave incidence, from which the series impedance $Z_{\mathrm{am}}$ of Fig. 4(b) could be extracted. ${ }^{35}$ Figure 5 shows the acoustic impedance of this membrane obtained from theory and full-wave simulation, and Table I reports the corresponding values for the elements of the approximate series resonant circuit of Fig. 4(b), for both theory and simulation. An excellent agreement is observed between theory

TABLE I. Acoustic mass and compliance for the considered membrane, calculated so that the approximate circuit of Fig. 4(b) fits the original impedance at the first resonance $f_{\mathrm{r}}$.

\begin{tabular}{lccc}
\hline \hline & $\begin{array}{c}m_{\mathrm{am}} \\
\left(\mathrm{kg} / \mathrm{m}^{4}\right)\end{array}$ & $\begin{array}{c}C_{\mathrm{am}} \\
\left(10^{-12} \mathrm{~m}^{3} / \mathrm{Pa}\right)\end{array}$ & $\begin{array}{c}f_{\mathrm{r}} \\
(\mathrm{Hz})\end{array}$ \\
\hline Theory, Eqs. (10) and (11) & 1296 & 17.42 & 1059 \\
Full-wave simulation & 1312 & 17.43 & 1052 \\
\hline
\end{tabular}




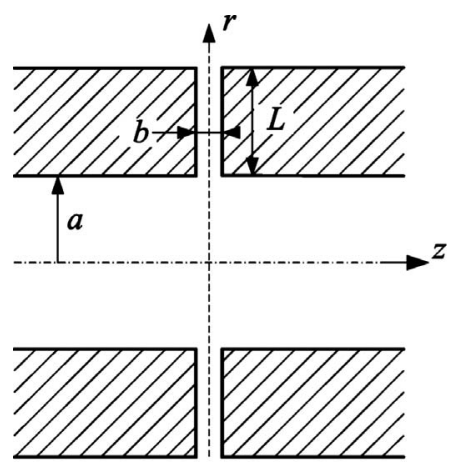

(a)

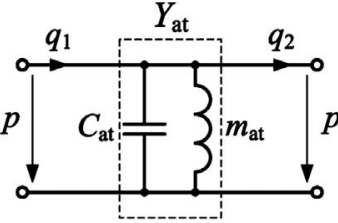

(b)

FIG. 6. Shorted radial stub connected to an acoustic circular waveguide. (a) Cut view. (b) Equivalent acoustic circuit.

and simulation, as well as between the original impedance and its "mass-compliance" circuit approximation. Figure 5 also confirms that the membrane impedance is dominated by a compliance below the resonance frequency $f_{\mathrm{r}}$.

\section{B. Realization of shunt acoustic masses with open channels}

\section{Description}

Shunt acoustic masses can be realized with short open tubes transversally connected to the main host waveguide. ${ }^{30}$ Such structures are often referred to as "stubs" in microwave engineering. This principle has been used in Ref. 21 to obtain an acoustic metamaterial with negative bulk modulus without using resonant elements such as Helmholtz resonators. ${ }^{17}$ We consider here a modified version of this structure that exhibits a perfect symmetry of revolution. It consists of a short radial waveguide of length $L$ and width $b$ connected to a main acoustic circular waveguide of radius $a$, as shown in Fig. 6(a). The considered stub is physically open at its termination, which approximately corresponds to a condition $p=0 .{ }^{29}$ As a result, the stub is approximately short circuited according to the chosen TL modeling, hence the name "shorted stub" that will be used thereafter.

\section{Theoretical modeling}

It can already be anticipated that such a stub represents an admittance $Y_{\text {at }}$ in parallel loading the TL representing the host waveguide, as shown in Fig. 6(b), since a certain amount of volume velocity is deviated into the stub, while the pressure does not experience any significant discontinuity. The admittance $Y_{\text {at }}$ corresponds to the input admittance of a short-circuited radial waveguide supporting only its axially symmetrical fundamental mode. Radial TL equations are similar to uniform TL equations with the particularity that propagation constant and characteristic impedance depend on the radial distance $r$, and therefore the solutions are expressed in terms of Bessel functions instead of exponential functions of the type $\exp ( \pm j k z)$. A detailed formulation of the radial TL theory developed in electromagnetics can be found in Ref. 36, which also applies to acoustic waves. Using this theory, the input acoustic impedance of a shorted radial stub, referred to here as $Z_{\mathrm{at}}\left(=1 / Y_{\mathrm{at}}\right)$, is given by

$$
Z_{\mathrm{at}}=j Z_{\mathrm{ac}}(a) \operatorname{tn}[k a, k(a+L)],
$$

where $\operatorname{tn}(x, y)$ is the small radial tangent function given by

$$
\operatorname{tn}(x, y)=\frac{J_{0}(x) N_{0}(y)-N_{0}(x) J_{0}(y)}{J_{1}(x) N_{0}(y)-N_{1}(x) J_{0}(y)},
$$

$N_{n}$ being the Bessel's function of the second kind of order $n$ and $Z_{\mathrm{ac}}(r)$ the acoustic characteristic impedance of the radial $\mathrm{TL}$ at a given radial distance $r$, given by

$$
Z_{\mathrm{ac}}(r)=\frac{Z_{\mathrm{c}}}{2 \pi r b} .
$$

It can be mentioned that the small radial tangent function "tn" is defined such that the expression for the input impedance is similar to that of a uniform TL, which is of the form $Z_{\text {at }}=j Z_{\text {ac }} \tan (k L)$.

The impedance in Eq. (12) exhibits a complex frequency dependence, but it can be approximated at low frequency by an acoustic mass, after

$$
Z_{\mathrm{at}} \approx j \omega m_{\mathrm{at} 0}, \quad \text { with } m_{\mathrm{at} 0}=\frac{\rho}{2 \pi b} \ln \left(1+\frac{L}{a}\right) .
$$

When the frequency increases the impedance quickly deviates from this simple model, which is thus accurate only for very small stubs compared to the wavelength. At higher frequency, the model can be improved by adding an acoustic compliance in shunt with the acoustic mass, as shown in Fig. 6(b). For a maximal accuracy of the model at a given frequency, it is useful to determine the $m_{\text {at }}$ and $C_{\text {at }}$ to fit the impedance, Eq. (12), and its frequency derivative at that frequency.

\section{Termination effects}

In practice, the stub termination is physically open and thus radiates into the surrounding medium. As a result, the impedance connected at the end of the stub is not exactly a short circuit but it exhibits a real part which accounts for radiation and an imaginary part representing the termination effects. For the narrow stubs considered in this work, the real part can be neglected and the remaining imaginary part is commonly ascribed to an increase in the stub length $\Delta L .^{29}$ Full-wave simulations are used to assist in the determination of the physical stub length $L$ corresponding to the desired effective length $L_{\mathrm{e}}=L+\Delta L$.

\section{Validation}

In order to validate the presented theoretical modeling, we consider a radial stub with $b=1 \mathrm{~mm}$ and $L=48.95 \mathrm{~mm}$ connected to an acoustic circular waveguide of radius $a=9.06 \mathrm{~mm}$. Figure 7 shows the input acoustic impedance of this stub and Table II reports the corresponding values for the elements of the approximate parallel resonant circuit of Fig. 6(b). The stub-loaded waveguide has been simulated with COMSOL MULTIPHYSICS and characterized in terms of reflection and transmission coefficients under plane-wave incidence, from which the shunt impedance $Z_{\text {at }}\left(=1 / Y_{\text {at }}\right)$ of Fig. 6(b) could be extracted. ${ }^{35}$ To compensate for the increase in 


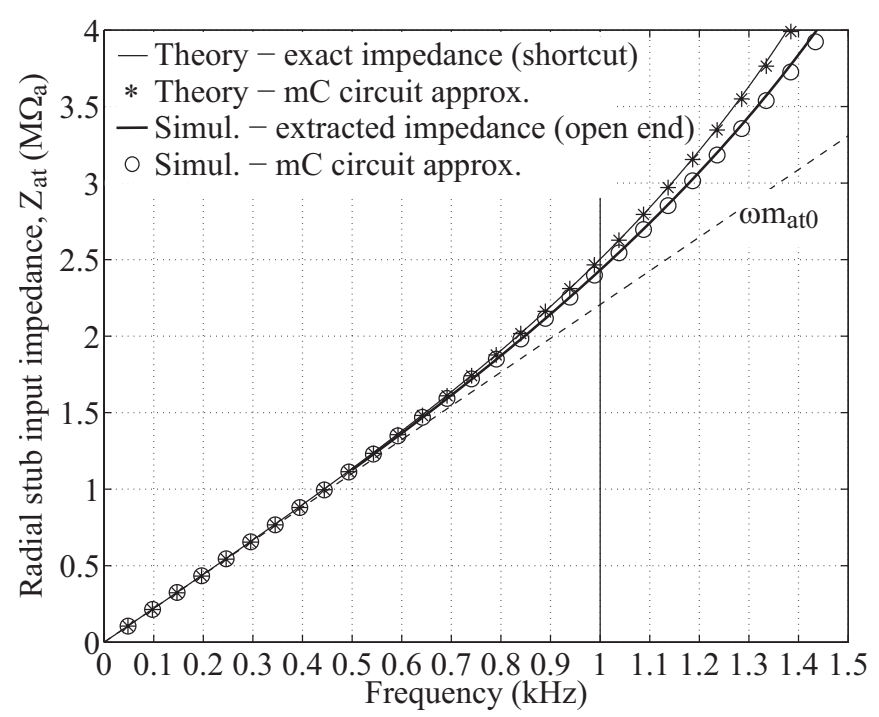

FIG. 7. Input acoustic impedance (imaginary part) of the considered radial stub. The Theory-exact impedance curve corresponds to Eq. (12) (ideal shortcut with $L=48.95 \mathrm{~mm}$ ), the Simul.extracted impedance curve is the impedance directly extracted from simulation results (open end with $L=42.67 \mathrm{~mm}$ ), and the two $\mathrm{mC}$ circuit approx. curves correspond to the circuit of Fig. 6(b) with the parameters given in Table II.

the effective length due to the open termination effects, the physical length of the stub has been decreased to approximately obtain the same acoustic mass as the perfectly shortcircuited stub. The resulting actual stub length is $L=42.67 \mathrm{~mm}$. The simulated performances of this stub are also shown in Fig. 7 and Table II.

A good agreement is observed between theory and fullwave simulation, as well as between the original impedance and its mass-compliance circuit approximation. It can be noted that the approximate expression (15) for the acoustic mass provides the value $m_{\text {at } 0}=351.1 \mathrm{~kg} / \mathrm{m}^{4}$, hence a difference inferior to $1 \%$ with the values given in Table II. Figure 7 also confirms that the impedance is dominated by an acoustic mass, as is the case for small stubs, although the shunt capacitance $C_{\text {at }}$ should be taken into account for precise modeling.

\section{Effect of viscous losses}

Narrow acoustic channels may be subject to viscous losses, a phenomenon which is assessed here for the considered stub. Viscous losses can be taken into account in the model of Fig. 6(b) by adding an acoustic resistance $R_{\text {at }}$ in series with the acoustic mass $m_{\mathrm{at}}$. Based on the Hagen-

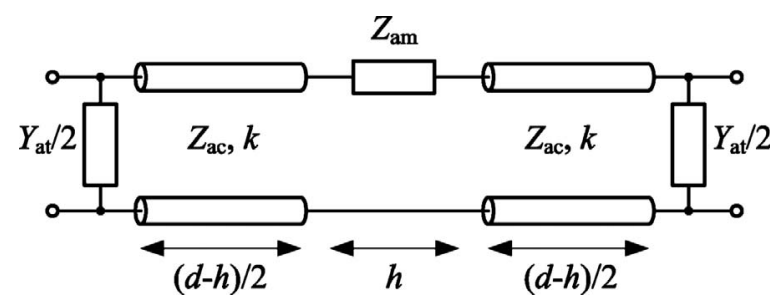

FIG. 8. Equivalent acoustic circuit for the symmetrical П-type unit cell of the considered acoustic CRLH TL.

Poiseuille assumption of laminar viscous fluid, the $R_{\text {at }}$ for a short radial stub can be approximated at low frequency by $^{29,34}$

$$
R_{\mathrm{at} 0}=\frac{6 \eta}{\pi b^{3}} \ln \left(1+\frac{L}{a}\right),
$$

where $\eta$ is the dynamic viscosity ( $\eta=18.6 \mu \mathrm{Pa}$ s for air). With this model of viscous fluid, the effective acoustic mass $m_{\mathrm{at} 0}^{\prime}$ is slightly higher than the original value $m_{\mathrm{at} 0}$ in Eq. (15), that is, $m_{\mathrm{at} 0}^{\prime}=1.2 m_{\mathrm{at} 0} \cdot{ }^{29}$ We further introduce the quality factor

$$
Q=\frac{\omega m_{\mathrm{at} 0}^{\prime}}{R_{\mathrm{at} 0}}=\frac{\omega \rho b^{2}}{10 \eta} .
$$

For the considered stub $(b=1 \mathrm{~mm})$ we have $Q=40$ at $1 \mathrm{kHz}$, therefore the resistance $R_{\text {at0 }}$ is 40 times smaller than the associated reactance $\omega m_{\text {at } 0}^{\prime}$. This value has been considered sufficiently large for neglecting, in a first approximation, the effect of viscous losses.

If viscous losses become a limiting factor for some specific applications, alternative stub topologies such as cylindrical tubes (one or several in parallel) shall be considered. Indeed, for the same targeted acoustic mass value, the required tubes diameter is less critical regarding this issue than the required width for radial stubs. For the concept validation presented here, whose goal is to demonstrate the capability of a stub to realize a shunt acoustic mass, the radial topology was chosen mostly for its symmetry of revolution since this property greatly simplifies the numerical modeling. Viscous losses are thus not a fundamental limitation, and we have provided here a simple way of addressing this issue in order to keep the associated effects below a prescribed level.

\section{CRLH TL MODELING AND DESIGN}

\section{A. Modeling}

Using the developments of Sec. III, the unit cell of the considered TL-based metamaterial introduced in Fig. 3 can

TABLE II. Acoustic mass and compliance for the considered radial stub, calculated so that the approximate circuit of Fig. 6(b) fits the original impedance at $1 \mathrm{kHz}$.

\begin{tabular}{lcc}
\hline \hline & $\begin{array}{c}m_{\mathrm{at}} \\
\left(\mathrm{kg} / \mathrm{m}^{4}\right)\end{array}$ & $\begin{array}{c}C_{\mathrm{at}} \\
\left(10^{-12} \mathrm{~m}^{3} / \mathrm{Pa}\right)\end{array}$ \\
\hline Theory (perfect shortcut, $L=48.95 \mathrm{~mm})$ & 348.3 & 9.138 \\
Full-wave simulation (open end, $L=42.67 \mathrm{~mm})$ & 350.1 & 6.897 \\
\hline \hline
\end{tabular}




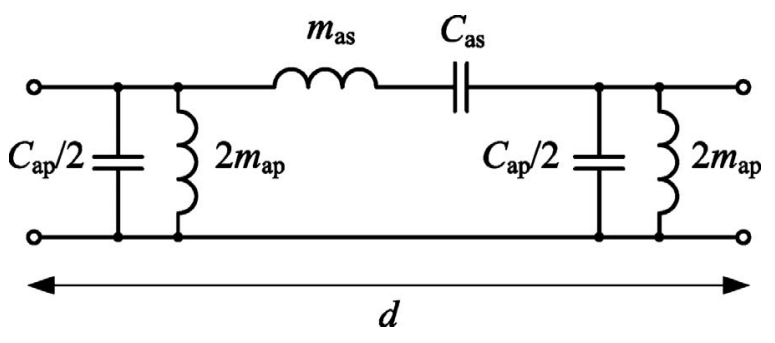

FIG. 9. Lumped element model for the symmetrical П-type unit cell of the considered acoustic CRLH TL (symmetrical version of the unit cell shown in Fig. 2).

be modeled by the circuit of Fig. 8. $Z_{\mathrm{am}}$ is the impedance of the membrane and $Y_{\text {at }}$ is the admittance of the radial stub, as described in Secs. III A and III B, respectively. The connections between these elements are modeled by TL sections of characteristic impedance $Z_{\mathrm{ac}}=Z_{\mathrm{c}} / S$ and phase constant $k$. The unit-cell boundaries are placed across the stubs positions in order to obtain a П-type network symmetrical unit cell. This is the reason why there is one half of $Y_{\mathrm{at}}$ at each side of the cell. The circuit of Fig. 8 can be further simplified as follows: (1) $Z_{\text {am }}$ and $Y_{\text {at }}$ are approximated by their masscompliance circuits of Figs. 4(b) and 6(b), respectively. (2) The effect of the TL sections is represented as shown in Fig. 1(a) by a series acoustic mass and a shunt acoustic compliance, denoted here $m_{\mathrm{aTL}}$ and $C_{\mathrm{aTL}}$. For both TL sections considered together, we have $m_{\mathrm{aTL}}=(\rho / S)(d-h)$ and $C_{\mathrm{aTL}}$ $=(S / K)(d-h)$. This approximate representation is accurate provided that the unit cell is small compared to the wavelength. (3) All the series and shunt elements are grouped together.

With these simplifications, we obtain the lumped element model of Fig. 9 with the parameters given by

$$
\left\{\begin{array} { c } 
{ m _ { \mathrm { as } } = m _ { \mathrm { am } } + m _ { \mathrm { aTL } } } \\
{ C _ { \mathrm { ap } } = C _ { \mathrm { at } } + C _ { \mathrm { aTL } } }
\end{array} \text { and } \left\{\begin{array}{c}
m_{\mathrm{ap}}=m_{\mathrm{at}} \\
C_{\mathrm{as}}=C_{\mathrm{am}} .
\end{array}\right.\right.
$$

\section{B. Characterization}

The periodic metamaterials considered in this work are characterized using the Bloch theory, whose basis are briefly recalled thereafter. ${ }^{37,38}$ Periodic structures support the propagation of eigenwaves often called Bloch waves. Looking at discrete locations corresponding to the boundaries of the cells $(z=n d, n \in \mathbb{Z})$, these waves exhibit a spatial dependence of the form $\exp \left( \pm \gamma_{\mathrm{B}} z\right)$. The quantity $\gamma_{\mathrm{B}}$ is referred to here as the Bloch propagation constant (i.e., the dispersion diagram). A characteristic impedance associated with the periodic structure can also be defined: it corresponds to the ratio between voltage and current at the boundaries of the cells for a purely traveling Bloch wave. This quantity is referred to here as the Bloch impedance $Z_{\mathrm{B}}$. Therefore, a periodic structure is fully characterized by its Bloch parameters $\gamma_{\mathrm{B}}$ and $Z_{\mathrm{B}}$, which are the periodic structure counterparts of the propagation constant and characteristic impedance defined for continuous TLs or waveguides.

The Bloch parameters $\gamma_{\mathrm{B}}$ and $Z_{\mathrm{B}}$ are related to the eigenvalues and eigenvectors of the transmission matrix (or $A B C D$
TABLE III. Values of the lumped elements associated with the proposed CRLH TL design [see Fig. 9 and Eq. (18)].

\begin{tabular}{lcc}
\hline \hline & $\begin{array}{c}m_{\mathrm{a}} \\
\left(\mathrm{kg} / \mathrm{m}^{4}\right)\end{array}$ & $\begin{array}{c}C_{\mathrm{a}} \\
\left(10^{-12} \mathrm{~m}^{3} / \mathrm{Pa}\right)\end{array}$ \\
\hline Membrane & $m_{\mathrm{am}}=1296$ & $C_{\mathrm{am}}=17.42$ \\
Radial stub & $m_{\mathrm{at}}=348.3$ & $C_{\mathrm{at}}=9.138$ \\
Host TL sections & $m_{\mathrm{aTL}}=156.1$ & $C_{\mathrm{aTL}}=63.60$ \\
Series elements & $m_{\mathrm{as}}=1452$ & $C_{\mathrm{as}}=17.42$ \\
Shunt elements & $m_{\mathrm{ap}}=348.3$ & $C_{\mathrm{ap}}=72.73$ \\
\hline \hline
\end{tabular}

matrix) of the unit cell, which links the voltage and current at one side of the cell to those at the other side. In the case of reciprocal and symmetrical unit cells, we obtain the relations $\gamma_{\mathrm{B}}=\operatorname{arcosh}\left(A_{\text {cell }}\right) / d$ and $Z_{\mathrm{B}}=\sqrt{B_{\text {cell }} / C_{\text {cell }}}$, where $d$ is the length of the unit cell. ${ }^{37-39}$ Application of this technique to the unit cell shown in Fig. 9 can be found in Ref. 25 or in Section 4.3 of Ref. 40, with all the corresponding expressions for $\gamma_{B}$ and $Z_{B}$ in function of the circuit elements. The refractive index $n$ can be simply deduced from the Bloch propagation constant using the relation $n=\gamma_{\mathrm{B}} /(j k)$.

\section{Design}

For the considered validation example, the following constraints have been imposed for the design: (1) The CRLH TL is balanced (closure of the stop band between the LH and RH bands ${ }^{25}$ ) with a transition frequency between the two bands $f_{0}=1 \mathrm{kHz}$. (2) The lattice constant $d$ should be small compared to the wavelength $\lambda$ in the host waveguide. In the present case, we have imposed $d / \lambda=0.1$ at $f_{0}$. (3) The Bloch impedance $Z_{\mathrm{B}}$ at $f_{0}$ should not be too far from the characteristic impedance of the host waveguide $\left(Z_{\mathrm{ac}}\right)$, in order to allow for a good matching to an external waveguide of comparable diameter.

The design has been performed using the lumped element model of Fig. 9 with the corresponding analytical expressions for the circuit elements (not detailed here). The resulting structure is the one shown in Fig. 3, with a lattice constant $d=34 \mathrm{~mm}$, a host waveguide radius $a=9.06 \mathrm{~mm}$, and the membranes and radial stubs are the one already described in Secs. III A 3 and III B 4, respectively. The associated values of the lumped elements involved in the model are reported in Table III. It can be observed that the balanced condition, which writes $m_{\mathrm{as}} C_{\mathrm{as}}=m_{\mathrm{ap}} C_{\mathrm{ap}},{ }^{25}$ is verified. It should be noticed here that perfect balance is difficult to achieve in practice since this property is very sensitive to any parameter fluctuation. Thus, although the structure is perfectly balanced according to the simplified model of Fig. 9, fine tuning of some parameters is often required to maintain the balanced condition for the real implemented structure. In the present case, this is the stub length $L$ that has been tuned for this purpose.

Using the detailed model of Fig. 8 with expressions (7) and (9) for $Z_{\mathrm{am}}$ and Eq. (12) for $Y_{\mathrm{at}}$, we found that $L$ had to be tuned from 48.95 to $46.80 \mathrm{~mm}$ (considering a perfect shortcut) in order to maintain the balanced condition. Further 

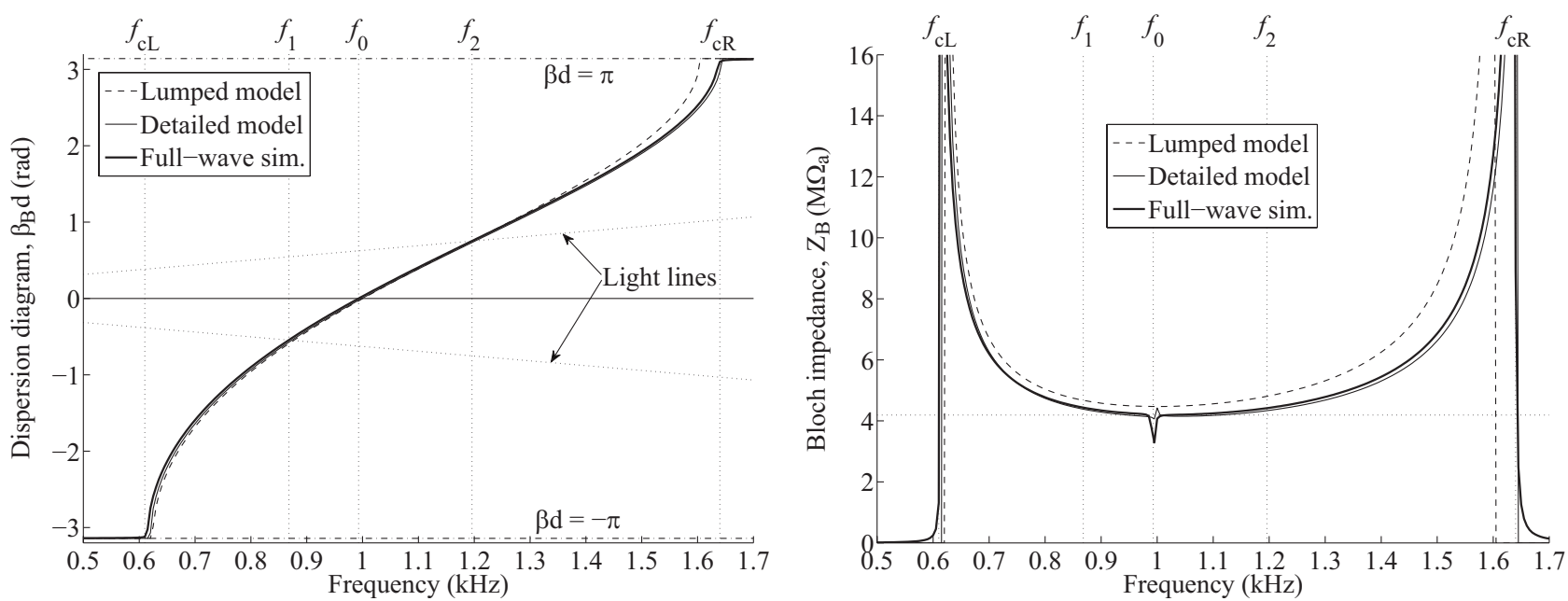

FIG. 10. Bloch parameters extracted from full-wave simulation results (COMSOL MULTIPHYSICS) and circuit models for the implemented CRLH TL. $f_{\mathrm{cL}}$ and $f_{\mathrm{cR}}$ are the LH and RH cut-off frequencies, respectively, $f_{0}$ is the transition frequency between the LH and RH bands, and $f_{1}$ and $f_{2}$ are the limits of the fast-wave band (see Sec. V D). The "light lines" correspond to the dispersion in air $(k= \pm \omega / c)$, by analogy with the terminology used in photonic crystals. The real part of $\gamma_{\mathrm{B}} d$ and the imaginary part of $Z_{\mathrm{B}}$ in the stopbands are not shown.

tuning based on full-wave simulations with the real open stub termination led to the physical stub length $L=43.50 \mathrm{~mm}$ for perfect balance.

\section{PERFORMANCES}

\section{A. Bloch parameters}

The Bloch parameters $\gamma_{\mathrm{B}}=\alpha_{\mathrm{B}}+j \beta_{\mathrm{B}}$ and $Z_{\mathrm{B}}$ have been calculated from the model of Fig. 8 , referred to here as the "detailed model," from the model of Fig. 9, referred to here as the "lumped model," and from full-wave simulation with COMSOL MULTIPHYSICS (see CAD model of Fig. 3). In the latter case, a single asymmetrical unit cell has been characterized in terms of reflection and transmission coefficients under plane-wave incidence, from which the Bloch parameters associated with the corresponding symmetrical П-type unit cell have been deduced. ${ }^{41}$ The results are shown in Fig. 10.

It can be observed on the dispersion diagram of Fig. 10(a) that the structure exhibits a negative refractive index band (opposite phase and group velocities) over a $50 \%$ relative bandwidth, from the LH cutoff $f_{\mathrm{cL}}=0.61 \mathrm{kHz}$ to the transition frequency $f_{0}=1 \mathrm{kHz}$, and a positive refractive index band from $f_{0}=1 \mathrm{kHz}$ to the RH cutoff $f_{\mathrm{cR}}=1.64 \mathrm{kHz}$. There is no band gap between these two bands (balanced condition), which results in a frequency with zero phase constant (and thus zero refractive index) and nonzero group velocity. An excellent agreement is observed between the full-wave results and the detailed circuit model (the curves are almost superimposed). The lumped element model also provides good results and can thus be efficiently used for preliminary designs.

The Bloch impedance at $f_{0}$ is $Z_{\mathrm{B} 0}=4.192 \mathrm{M} \Omega_{\mathrm{a}}$ (horizontal line in the $Z_{\mathrm{B}}$ plot). The structure can thus be matched at that frequency to an acoustic circular waveguide of characteristic acoustic impedance $Z_{\mathrm{ace}}=Z_{\mathrm{B} 0}$, which corresponds to a radius $a_{\mathrm{e}}=5.54 \mathrm{~mm}\left[Z_{\mathrm{ace}}=Z_{\mathrm{c}} /\left(\pi a_{\mathrm{e}}^{2}\right)\right]$. Moreover, the Bloch impedance around the transition frequency $f_{0}$ is smoothly varying with frequency, which is favorable for wideband matching.

\section{B. Equivalent medium parameters}

Although the periodic structure is completely characterized by its Bloch parameters $\gamma_{\mathrm{B}}$ and $Z_{\mathrm{B}}$, equivalent medium parameters can also be derived by analogy with a plane wave propagating in an equivalent fluid. This formal analogy consists in mapping the impedance of the series branch of the unit-cell circuit with the impedance associated with an equivalent mass density $\rho_{\mathrm{eq}}$ and the admittance of the shunt branch with the one associated with an equivalent bulk modulus $K_{\text {eq. }}$. For a CRLH TL, a simple comparison of the circuits of Figs. 1(a) and 2 yields

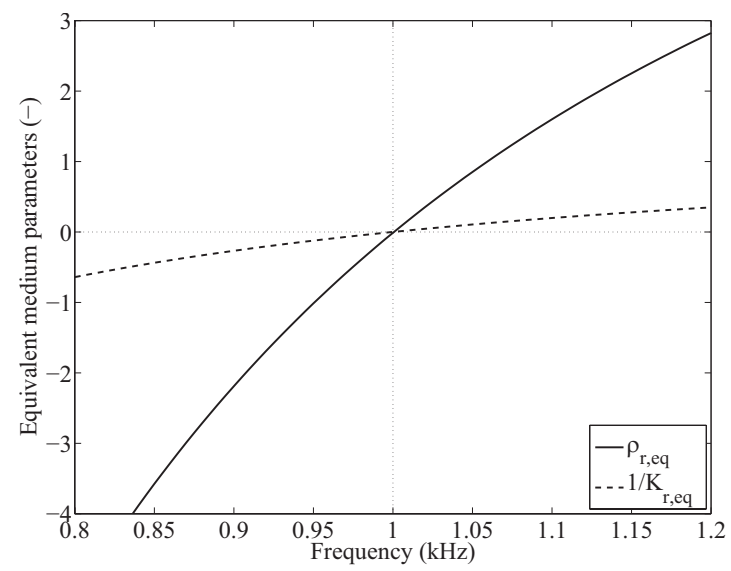

FIG. 11. Equivalent mass density and bulk modulus (relative values) for the considered CRLH TL around the transition frequency, calculated from the lumped element model of Fig. 9. 


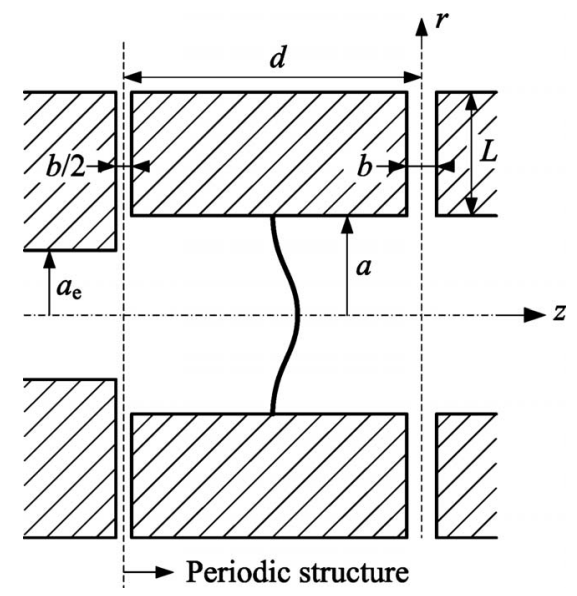

FIG. 12. Modified half-width stub at the periodic structure termination (idem at the other side).

$$
\left\{\begin{array} { l } 
{ \rho _ { \mathrm { eq } } = \frac { Z _ { \mathrm { as } } } { j \omega } \cdot \frac { S } { d } } \\
{ K _ { \mathrm { eq } } ^ { - 1 } = \frac { Y _ { \mathrm { ap } } } { j \omega } \cdot \frac { 1 } { S d } }
\end{array} \text { with } \left\{\begin{array}{l}
Z_{\mathrm{as}}=j \omega m_{\mathrm{as}}+\frac{1}{j \omega C_{\mathrm{as}}} \\
Y_{\mathrm{ap}}=j \omega C_{\mathrm{ap}}+\frac{1}{j \omega m_{\mathrm{ap}}} .
\end{array}\right.\right.
$$

Therefore, this is as if the same host waveguide was filled with an homogeneous medium characterized by $\rho_{\mathrm{eq}}$ and $K_{\mathrm{eq}}$. For convenience, we define the corresponding relative quantities $\rho_{\mathrm{r}, \mathrm{eq}}=\rho_{\mathrm{eq}} / \rho$ and $K_{\mathrm{r}, \mathrm{eq}}^{-1}=K_{\mathrm{eq}}^{-1} / K^{-1}$, which are plotted in Fig. 11 for the considered CRLH TL. It can be observed that they exhibit a nonresonant behavior with negative values over a relatively large bandwidth below the transition frequency $f_{0}$. It confirms that a series compliance is needed to make the mass density become negative, as the shunt mass does for the bulk modulus. This motivates the developments and use of circuit models as an efficient tool to achieve negative parameters.

This analogy only makes sense provided that the lattice constant $d$ is small compared to the wavelength in the equivalent medium, thus around the transition frequency, where this wavelength approaches infinity. Outside this band, periodicity effects such as the occurrence of the stopbands below $f_{\mathrm{cL}}$ and above $f_{\mathrm{cR}}$ begin to play an important role, which prevents a meaningful effective-medium description.

\section{Scattering parameters for a 10-cell-long structure}

The performances of the proposed CRLH TL are further validated by considering a 10-cell-long structure characterized in terms of reflection and transmission coefficients $\rho$ and $\tau$, respectively (or scattering parameters), under plane-wave incidence from an external waveguide of radius $a_{\mathrm{e}}=5.54 \mathrm{~mm}$ (see Sec. V A). As the unit cell is symmetrical of the $\Pi$ type (see Fig. 8 or 9), the structure should be terminated at both sides with an admittance $Y_{\text {at }} / 2$, or equivalently an acoustic mass $2 m_{\text {at }}$ and an acoustic compliance $C_{\text {at }} / 2$. This is approximately achieved using a stub of half width $b / 2$, as shown in Fig. 12.

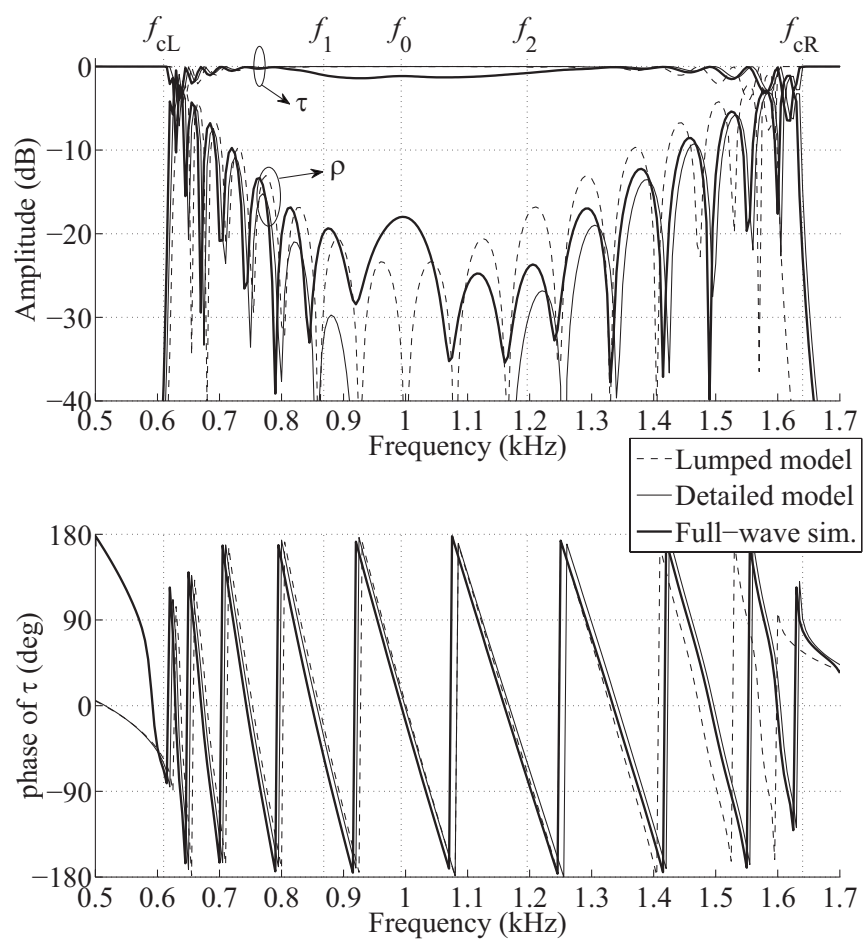

FIG. 13. Reflection $\rho$ and transmission $\tau$ coefficients for a 10cell-long CRLH TL. The phase of $\rho$ is not shown for clarity.

The resulting scattering parameters obtained from fullwave simulations and circuit models are shown in Fig. 13. As imposed in the design, the CRLH TL is well matched over a large part of its passband $(|\rho|<-10 \mathrm{~dB}$ from about 0.7 to $1.45 \mathrm{kHz}$ ) and the transmission phase is 0 around 1 $\mathrm{kHz}$. Globally, a good agreement is observed between fullwave and circuit model results, the detailed model being slightly more accurate with respect to the full-wave model than the lumped model, as expected. However, it can be observed that the simulated reflection coefficient $\rho$ is not as low as determined using the detailed model around the transition frequency $f_{0}$. This is attributed to radiation, as explained in Sec. V D, a phenomenon which cannot be accounted for in the considered circuit models since they only involve lossless elements.

\section{Radiation losses}

As all the materials in the proposed CRLH TL have been considered as lossless in the full-wave simulations, the losses, if any, can only be due to radiation from the stubs apertures. To assess the importance of this phenomenon, the "efficiency" defined as $\eta=|\rho|^{2}+|\tau|^{2}$ has been calculated from the full-wave simulation results of the 10-cell structure and is plotted in Fig. 14. If no losses are present, we should have $\eta=1$. It can be observed that radiation losses mostly occur between $f_{1}$ and $f_{2}$, where about $25 \%$ of the power is lost by radiation. This frequency range coincides with the so-called fast-wave band observed in the dispersion diagram of Fig. $10(\mathrm{a})$, in which the phase velocity in the periodic structure is faster than the velocity of acoustic waves in the surrounding medium (air) (light lines). This effect is analogous to Cher- 


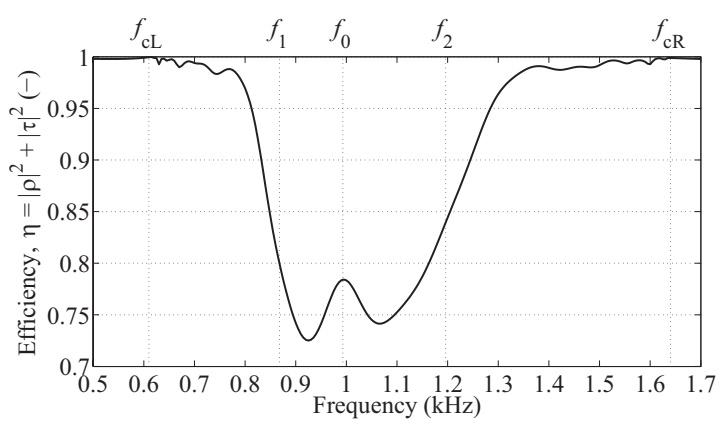

FIG. 14. Efficiency for the 10-cell CRLH TL calculated from full-wave simulation.

enkov radiation of a charged particle which exceeds the speed of light in a medium. This peculiar feature for this type of metamaterials has been extensively exploited in microwave antenna engineering for the development of a different type of leaky-wave antennas with full-space scanning capabilities, from backfire (at $f_{1}$ ) to endfire (at $f_{2}$ ), including broadside (at $f_{0}$ ). 2,24,25 The proposed structure thus represents the analog for acoustic waves of these antennas, thereby opening the door for the investigation of directive acoustic sensors or sources based on this principle. Such structures can also be seen as arrays of weakly radiating apertures fed with the required phase shift by the periodic structure itself.

It can be finally mentioned that, although the considered circuit models cannot directly account for radiation losses due to their lossless nature, they can predict at which frequency radiation will take place thanks to an accurate estimation of the dispersion diagram. Indeed, the latter is not significantly affected by losses, provided that the amount of losses per cell remains small.

\section{CONCLUSION}

Circuit-theory concepts have been efficiently used to conceptualize and design an acoustic nonresonant TL-based metamaterial. The particularity resides in using membranes to implement the required series compliances, whereas the shunt acoustic masses have been achieved with transversally connected open channels. This structure presents interesting properties in, at least, two regards. First, it exhibits a negative refractive index over almost one octave $(0.6-1 \mathrm{kHz})$, which is considerably larger than what can be achieved with locally resonant acoustic metamaterials. Second, the structure exhibits a balanced CRLH response, i.e., no band gap exists between the positive and negative index frequency bands which touch at a particular frequency with zero refractive index and nonzero group velocity. Moreover, the lattice constant is ten times smaller than the wavelength, which largely justifies an effective-medium description. The aforementioned performances have been confirmed by full-wave simulations in terms of dispersion diagram, Bloch impedance, and reflection and transmission coefficients.

The radiation properties of this periodic structure have also been investigated. They point out to interesting applications, in which the structure could be used as a directive acoustic sensor or source with enhanced beaming characteristics, by analogy with the metamaterial leaky-wave antennas developed in microwave engineering. ${ }^{2,24,25}$

The developed structure might also find applications in subwavelength resonant cavities based on phase compensation between negative and positive index media. ${ }^{42}$ Further work should aim at the investigation of two-dimensional structures based on the same principle. Here, targeted applications are wideband and well-matched negative index acoustic lenses for acoustic imaging applications. The specific zero refractive index frequency may also find very interesting applications ${ }^{43}$ and similar structures can also be adapted to cloaking. ${ }^{44,45}$ Indeed, although cloaking does not explicitly require negative refractive index media, the proposed structures based on membranes and/or stubs can also be operated in the band where they exhibit a positive but less than one refractive index. In summary it is hoped that the methods developed in this paper will pave the way for experimental validation of these exciting properties on real prototypes, that should be obtained through a one-pass, pitfalldevoid design process.

\footnotetext{
*frederic.bongard@a3.epfl.ch

†herve.lissek@epfl.ch

${ }^{1}$ N. Engheta and R. W. Ziolkowski, Metamaterials: Physics and Engineering Explorations (Wiley-Interscience and IEEE Press, Hoboken, NJ, 2006).

${ }^{2}$ G. V. Eleftheriades and K. G. Balmain, Negative-Refraction Metamaterials: Fundamental Principles and Applications (Wiley-Interscience and IEEE Press, Hoboken, NJ, 2005).

${ }^{3}$ M. Ambati, N. Fang, C. Sun, and X. Zhang, Phys. Rev. B 75, 195447 (2007).

${ }^{4}$ J. B. Pendry, Phys. Rev. Lett. 85, 3966 (2000).

${ }^{5}$ S. Yang, J. H. Page, Z. Liu, M. L. Cowan, C. T. Chan, and P. Sheng, Phys. Rev. Lett. 93, 024301 (2004).

${ }^{6}$ X. Zhang and Z. Liu, Appl. Phys. Lett. 85, 341 (2004).

${ }^{7}$ M. Ke, Z. Liu, C. Qiu, W. Wang, J. Shi, W. Wen, and P. Sheng,
}

Phys. Rev. B 72, 064306 (2005).

${ }^{8}$ M. Ke, Z. Liu, Z. Cheng, J. Li, P. Peng, and J. Shi, Solid State Commun. 142, 177 (2007).

${ }^{9}$ P. A. Deymier, B. Merheb, J. O. Vasseur, A. Sukhovich, and J. H. Page, Rev. Mex. Fis. 54, 74 (2008).

${ }^{10}$ L. Fok, M. Ambati, and X. Zhang, MRS Bull. 33, 931 (2008).

${ }^{11}$ C. T. Chan, J. Li, and K. H. Fung, J. Zhejiang Univ., Sci. 7, 24 (2006).

${ }^{12}$ J. Li and C. T. Chan, Phys. Rev. E 70, 055602 (2004).

${ }^{13}$ Y. Ding, Z. Liu, C. Qiu, and J. Shi, Phys. Rev. Lett. 99, 093904 (2007).

${ }^{14}$ Z. Liu, X. Zhang, Y. Mao, Y. Y. Zhu, Z. Yang, C. T. Chan, and P. Sheng, Science 289, 1734 (2000).

${ }^{15}$ Z. Liu, C. T. Chan, and P. Sheng, Phys. Rev. B 71, 014103 (2005). 
${ }^{16}$ H. Larabi, Y. Pennec, B. Djafari-Rouhani, and J. O. Vasseur, Phys. Rev. E 75, 066601 (2007).

${ }^{17}$ N. Fang, D. Xi, J. Xu, M. Ambati, W. Srituravanich, C. Sun, and X. Zhang, Nature Mater. 5, 452 (2006).

${ }^{18}$ Z. G. Wang, S. H. Lee, C. K. Kim, C. M. Park, K. Nahm, and S. A. Nikitov, J. Phys.: Condens. Matter 20, 055209 (2008).

${ }^{19}$ Y. Cheng, J. Y. Xu, and X. J. Liu, Appl. Phys. Lett. 92, 051913 (2008).

${ }^{20}$ X. Hu, K.-M. Ho, C. T. Chan, and J. Zi, Phys. Rev. B 77, 172301 (2008).

${ }^{21}$ S. H. Lee, C. M. Park, Y. M. Seo, Z. G. Wang, and C. K. Kim, J. Phys.: Condens. Matter 21, 175704 (2009).

${ }^{22}$ M. S. Kushwaha, A. Akjouj, B. Djafari-Rouhani, L. Dobrzynski, and J. O. Vasseur, Solid State Commun. 106, 659 (1998).

${ }^{23}$ T. Koschny, P. Markos, D. R. Smith, and C. M. Soukoulis, Phys. Rev. E 68, 065602 (2003).

${ }^{24}$ A. Lai, C. Caloz, and T. Itoh, IEEE Microw. Mag. 5, 34 (2004).

${ }^{25} \mathrm{C}$. Caloz and T. Itoh, Electromagnetic Metamaterials: Transmission Line Theory and Microwave Applications (WileyInterscience and IEEE Press, Hoboken, NJ, 2006).

${ }^{26} \mathrm{G}$. V. Eleftheriades, A. K. Iyer, and P. C. Kremer, IEEE Trans. Microwave Theory Tech. 50, 2702 (2002).

${ }^{27}$ S. H. Lee, C. M. Park, Y. M. Seo, Z. G. Wang, and C. K. Kim, Phys. Lett. A 373, 4464 (2009).

${ }^{28}$ S. H. Lee, C. M. Park, Y. M. Seo, Z. G. Wang, and C. K. Kim, Phys. Rev. Lett. 104, 054301 (2010).

${ }^{29}$ M. Rossi, Acoustics and Electroacoustics (Artech House, Norwood, MA, 1988).

${ }^{30}$ L. E. Kinsler, A. U. Frey, A. B. Coppens, and J. V. Sanders, Fundamentals of Acoustics (Wiley, New York, 1982).
${ }^{31}$ This corresponds to average conditions with a static pressure of $980 \mathrm{hPa}$ and a temperature of $15^{\circ} \mathrm{C}$.

${ }^{32}$ Z. Yang, J. Mei, M. Yang, N. H. Chan, and P. Sheng, Phys. Rev. Lett. 101, 204301 (2008).

${ }^{33}$ P. M. Morse and K. U. Ingard, Theoretical Acoustics (Princeton University Press, Princeton, NJ, 1986).

${ }^{34}$ Z. Skvor, Vibrating Systems and their Equivalent Circuits (Elsevier, New York, 1991).

${ }^{35}$ Details on the method used to extract lumped element models for waveguide discontinuities can be found in Sec. 4.4.3 of Ref. 40.

${ }^{36}$ N. Marcuwitz, Waveguide Handbook (McGraw-Hill, New York, 1951).

${ }^{37}$ R. E. Collin, Foundations for Microwave Engineering (McGrawHill, New York, 1992).

${ }^{38}$ D. M. Pozar, Microwave Engineering (Addison-Wesley, New York, 1990).

${ }^{39}$ J. Perruisseau-Carrier and A. K. Skrivervik, IEEE Trans. Microwave Theory Tech. 54, 1582 (2006).

${ }^{40}$ F. Bongard, Ph.D. thesis, Ecole Polytechnique Fédérale de Lausanne (EPFL), 2009.

${ }^{41}$ The postprocessing operation allowing to calculate the Bloch parameters associated with the symmetrical unit cell from the knowledge of the asymmetrical one is not detailed here but more details can be found in Sec. 4.2.8 of Ref. 40.

${ }^{42}$ N. Engheta, IEEE Antennas Wireless Propag. Lett. 1, 10 (2002).

${ }^{43}$ R. W. Ziolkowski, Phys. Rev. E 70, 046608 (2004).

${ }^{44}$ S. A. Cummer, B.-I. Popa, D. Schurig, D. R. Smith, J. B. Pendry, M. Rahm, and A. Starr, Phys. Rev. Lett. 100, 024301 (2008).

${ }^{45}$ H. Chen and C. T. Chan, Appl. Phys. Lett. 91, 183518 (2007). 